Pak. j. sci. ind. res. Ser. B: biol. sci. 201356 (2) 76-81

\title{
Pharmacokinetic Modelling of Methotrexate from Routine Clinical Data in Patients with Acute Lymphoblastic Leukemia
}

\author{
Nadia Jebabli*, Hanen El Jebari, Emna Gaïes, Issam Salouage, Sameh Trabelsi, Imen Hamza, \\ Anis Klouz and Mohamed Lakhal \\ Laboratory of Clinical Pharmacology, Centre National de Pharmacovigilance, Tunisia
}

(received April 25, 2011; revised October 2, 2012; accepted November 26, 2012)

\begin{abstract}
Pharmacokinetic modelling was performed in NONMEM (version 6.1) using a dataset including 273 patients (aged 2 to 23 years) who received high-dose MTX ( $5 \mathrm{~g} / \mathrm{m}^{2}$ per course) in longterm treatment. Total 2582 methotrexate plasma concentrations were performed by fluorescence polarisation immunoassay (FPIA). A three compartment open model with elimination from the central compartment described the pharmacokinetics of methotrexate. The most important covariates affecting the disposition of methotrexate were age (age, year), body weight $(\mathrm{BW}, \mathrm{kg})$, and creatinine clearance $\left(\mathrm{CLR}, \mathrm{lh}^{-1}\right)$. The final model with exponential disposition of MTX was clearance $\left(\mathrm{CL}, \mathrm{lh}^{-1}\right)=(6.11+$ $\left.\mathrm{WT}^{*} 6.7310^{-2}\right)+\left(1.0810^{-4} * \mathrm{CLR}\right) * \operatorname{EXP}\left(1.9510^{-1}\right),(\mathrm{V}, 1)=10,8+\left(\mathrm{AGE}^{*} 9.310^{-2}\right) * \operatorname{EXP}\left(9.110^{-1}\right)$, $\mathrm{Q}\left(\mathrm{lh}^{-1}\right)=2.0410^{-3} * \mathrm{WT}$. Pharmacokinetic parameters $(\% \mathrm{CV})$ in this study were $\mathrm{CL}, 8.72 \mathrm{lh}^{-1}(44 \%)$; V1, 17.49 1 (95\%); V2, 6.048 1 (56\%); V3, 0.015 1(52\%). The model predictions in the qualification group were found to have no bias and satisfactory precision
\end{abstract}

Keywords: population pharmacokinetics, methotrexate, acute lymphoblastic leukaemia, NONMEM

\section{Introduction}

Methotrexate (MTX) with folinic acid rescue is widely used in the treatment of leukaemia. However, high dose MTX has been proven to cause substantial toxicity and have high intra-and inter-patient variability.

The MTX is characterized by a narrow therapeutic window combined with high-and inter-patient variability (Dupuis et al., 2006). It has been demonstrated that HDMTX toxicity is directly linked to its exposure time and plasma concentration (Balloy et al., 2007). Elimination of MTX is prolonged in patients with renal impairment or third space fluid collections due to a slow redistribution from this extravascular fluid accumulation.

Population pharmacokinetic analysis is a useful tool for identification of sources of pharmacokinetic variability during anticancer drug development and can aid the design of alternative dosing regimens to enhance their efficacy and safety.

Objective of this study was to develope and validate a population pharmacokinetics model of MTX in Tunisian patients with acute lymphoblastic leukaemia (ALL) (aged from 2 to 23 years). Clinical covariates have been described that influence MTX pharmacokinetic for predicting optimal dose to reduce MTX toxicity.

\footnotetext{
*Authour for correspondence; E-mail: nadiascience@voila. fr
}

\section{Materials and Methods}

Patients. Two hundred and seventy three patients (aged 2 - 23 years, median 13 years) who received high-dose MTX therapy (1-4 courses of chemotherapy) have been studied. The disease treated was ALL. They were enrolled at the time of diagnosis in the European Organization for Research and Treatment of Cancer (EORTC) protocol 58951 (Falkenrodt et al., 1994) approved by an institutional review committee for clinical trials.

Data collection. Blood samples were collected by prospective chart review including: age, gender, weight, body surface area (BSA), significant delay in MTX elimination defined as MTX concentration at $24 \mathrm{~h}$ after the end of infusion $\left(\mathrm{Cp}_{24} \mathrm{~h}\right)>5 \mu \mathrm{mol} / \mathrm{L}$, and/or MTX concentration $48 \mathrm{~h}$ after the end of the infusion $\left(\mathrm{Cp}_{48 \mathrm{~h}}\right)$ $>0.5 \mu \mathrm{mol} / \mathrm{L}$.

MTX administration. Urine alkalinisation was performed by administration of $1.4 \%$ sodium bicarbonate, $10 \mathrm{~mL} / \mathrm{kg}, 2 \mathrm{~h}$ prior to the start of MTX infusion. Intravenous hydration (1.4\% sodium bicarbonate $(1 / 3)$ and $5 \%$ glucose solution with $2 \mathrm{~g} / \mathrm{L} \mathrm{KCl} \mathrm{(2/3),} 24$ $\mathrm{mL} / \mathrm{m}^{2} / 24 \mathrm{~h}$ ) was administered from the start of the infusion up to $72 \mathrm{~h}$. The dose MTX $\left(5000 \mathrm{mg} / \mathrm{m}^{2}\right)$ was given as a loading dose $\left(1600 \mathrm{mg} / \mathrm{m}^{2}\right.$ over $0.5 \mathrm{~h}$ in 250 $\mathrm{mL}$ of $5 \%$ glucose), followed by the rest of the dose $\left(6400 \mathrm{mg} / \mathrm{m}^{2}\right.$ over $23.5 \mathrm{~h}$ in $500-1000 \mathrm{~mL}$ of $5 \%$ glucose). 
Urine $\mathrm{pH}$ was checked after every $6 \mathrm{~h}$ and a dose of $1.4 \%$ sodium bicarbonate, $6 \mathrm{~mL} / \mathrm{kg} \mathrm{IV}$, was administered if $\mathrm{pH}<7$. Folinic acid, at the dose of $12 \mathrm{mg} / \mathrm{m}^{2}$ IVwas administered to adjust to MTX concentrations according to protocol guidelines, at $36 \mathrm{~h}$ after the initiation of MTX infusion and repeated every $6 \mathrm{~h}$ until MTX concentration was $<0.02 \mu \mathrm{mol} / \mathrm{L}$.

Sample analysis. For each patient, the blood samples were collected in dry tubes protected from light 24,48 , $72,96 \mathrm{~h}$ at the end of infusion and rapidly centrifuged at $2500 \mathrm{~g}$ and stored at $-20^{\circ} \mathrm{C}$ until simultaneous analysis. MTX concentrations were measured with ABBOTT $\mathrm{TD}_{\mathrm{X}}$ fluorescence polarization immunoassay (FPIA). The detection limit of the assay was $0.01 \mu \mathrm{mol} / \mathrm{L}$. Cross reactivity with the main circulating metabolite, i.e. 7-hydroxy MTX (7-OH-MTX), was less than 1.5\%. Cross reactivity with 2.4 diamino-N-methyl-pteroic acid was as high as $44 \%$; but this metabolite produced in the gut is usually undetectable in plasma samples and therefore could not interfere with the assay (Colom et al., 2009). The assay by FPIA was highly sensitive and rapid enough to measure plasma MTX concentration.

Pharmacokinetics analysis. Population pharmacokinetic modelling was applied using NONMEM (version 6.1) to estimate pharmacokinetic parameters, intersubject and residual variability (random effects) in terms of patients specific information such as age or body weight (fixed effects). All analyses were performed with the first-order conditional estimation method with interaction. Graphical diagnostics and comparison of competing models using the objective function values (OFV) in the likelihood ratio test guided the model development.

Statistical model. The choice of the structural (or pharmacokinetic) model was based on a comparison of two and three compartment models with first order input and first order elimination from the central compartment. Analysis of model diagnostics favoured selection of the three compartment model. Therefore, a three open compartmental model disposition of methotrexate was described in terms of clearance $(\mathrm{Cl})$, volume V1 of central compartment, volume V2 and V3 of the peripheral compartments, the intercompartmental clearance $\mathrm{Q}_{2}, \mathrm{Q}_{3}$ between the central and peripheral compartments, and the elimination constant $\mathrm{K}_{10}$ from the central compartment. (PREDPP subroutine ADVAN11 TRANS4 in NONMEM).
To estimate the pharmacokinetic parameters of methotrexate in the investigated population, the following models were used to describe the intersubject variability in cleareance:

$\mathrm{Cl}_{\mathrm{i}}=\mathrm{Clpop}^{*} \mathrm{e}^{\eta_{\mathrm{JCL}, \mathrm{i}}}$

Where, $\mathrm{Cl}_{\mathrm{i}}$ is the plasma clearance of MTX from the ' $\mathrm{j}^{\text {th }}$ patient; $\mathrm{Cl}$ pop is the population mean value of $\mathrm{Cl}$ or a known function that describes the expected value of $\mathrm{Cl}_{\mathrm{j}}$ as a function of individual specific covariates, such as age, body weight, gender, etc, and the vector of population average parameters. $\eta_{\mathrm{JCL}, \mathrm{i}}$ is betweenpatient variability (BPV) and inter-occasion variability were modelled exponentially assuming a log-normal distribution. Residual error (intra individual variability) in the concentration was modelled with exponential error structure as follows:

$\mathrm{Cij}=\mathrm{F}^{*} \exp \left(\varepsilon_{1, \mathrm{i}, \mathrm{j}}\right)$

Where, $\mathrm{Cij}$ is the $\mathrm{j}^{\text {th }}$ methotrexate serum concentration observation in the $\mathrm{i}^{\text {th }}$ individual, Fij is the $\mathrm{j}^{\text {th }}$ model predicted concentration in the $\mathrm{i}^{\text {th }}$ individual, and $\varepsilon_{\mathrm{i}, \mathrm{j}}$ are the residual variability error terms and are assumed normally distributed with mean 0 and variance $\sigma^{2}$ which is sometimes written in shorthand as $\varepsilon=\mathrm{N}\left(0, \sigma^{2}\right)$.

Development of covariate model. The regression model describes the relationship between a pharmacokinetic parameter and a covariate. A graphical approach to exploratory data analysis can be useful tool in elucidating the presence of these relationships. An initial screening with Excel $^{\circ}$ software was performed. The initial screening gives a first impression of the relative importance of several covariates and pharmacokinetic parameters. To carry out this preliminary step, individual estimates of clearance were first obtained and subsequently the significance of each possible covariate in affecting the parameter was evaluated. After the initial screening step, with the estimates of the individual $\mathrm{Cl}$ values treated as 'data', a regression model was derived with stepwise regression. This step corresponds to the classical regression problem of variable selection. The influence of age (AGE), body weight (BW), gender (GEN, $0=$ male, $1=$ female), creatinine clearance (CLR) was observed.

Development of a regression model is an attempt to maximize the predictive ability of the model without sacrificing parsimony. To achieve this objective, 
covariates were first screened by individually testing each covariate alone in the base pharmacostatistical model to determine which ones significantly improves the ability of the model to predict the observed concentration-time profile. The difference in the objective function value (- $2 * \log$ likelihood) between two hierarchical models, defined as the log likelihood difference (LLD), is asymptomatically chi squared $\left(\chi^{2}\right)$ distributed with degrees of freedom equal to the difference in the number of parameters between the two models. During the covariate screening step, addition of one parameter to the model had to effect a LLD of at least 3.84 to achieve the desired level of significance of $\alpha=0.05$ $(p<0.05)$ from chi squared distribution with one degree of freedom. Traditional methods of model building reported in the literature assemble all significant covariates into an intermediate full model that is then subjected to backwards elimination procedure to produce a parsimonious model (Combe et al., 1995).

Qualification of the model. To evaluate the performance of the final model in predicting MTX concentrations, a second group of 50 patients treated with MTX was studied. The demographic data of the evaluation group is shown in Table 1. The measured concentrations of MTX in these patients were compared with the corresponding predicted values obtained using the final population pharmacokinetic model, patients' covariates and dosing information. Predictive performance of the model was assessed by calculating the mean error (predicted-observed concentration) and its 95\% confidence interval (CI) as an estimate of bias, and the root mean squared prediction error and $95 \% \mathrm{CI}$ as an estimate of precision. CIs including the value zero were considered unbiased (Sheiner and Ludden, 1992).

The final model was subjected to a bootstrap analysis (1,000 replicates) using R software-package (version 2.9.2). Table 2 summarizes the results presented as medians and $95 \%$ confidence intervals $\left(2.5^{\text {th }}\right.$ and $97.5^{\text {th }}$ percentiles). Bootstrap medians and parameter estimates from the original dataset were reasonably similar and indicated acceptable precision.

\section{Results and Discussion}

Patients. Pharmacokinetic data were available for 273 children and young adults with ALL receiving high dose of MTX $\left(5 \mathrm{~g} / \mathrm{m}^{2}\right)$. Patient characteristics are outlined in Table 1.

Pharmacokinetic model. Analyses of residual plots from the two and three compartment model fits were compared. The three compartment model provides a superior fit to the data compared to the two compartment model fit (LLD increased by 128 , statistically significant $\mathrm{p}<0.05)$. Potential explanatory covariables might include patient's age, weight, gender, height, and creatinine clearance. The change in the NONMEM objective function produced by the inclusion of a covariate term was used to compare alternative models. Finally, accepted covariates were added to the model and the population pharmacokinetic parameters were estimated. To

Table 1. Patient characteristics

\begin{tabular}{llll}
\hline \hline Parameters & $\begin{array}{l}\text { Population } \\
\text { analysis } \\
(\text { Mean } \pm \text { SD) }\end{array}$ & $\begin{array}{l}\text { Qualification } \\
\text { analysis } \\
(\text { Mean } \pm \mathrm{SD})\end{array}$ & $\begin{array}{l}\text { Abrev- } \\
\text { iation }\end{array}$ \\
\hline $\begin{array}{l}\text { Age (years) } \\
\text { Body weight } \\
(\mathrm{kg})\end{array}$ & $13.74 \pm 8.57$ & $10.59 \pm 5.12$ & Age \\
$\begin{array}{l}\text { Body surface } \\
\left.\mathrm{m}^{2}\right)\end{array}$ & $42.40 \pm 18.9$ & $35.52 \pm 19.31$ & WT \\
$\begin{array}{l}\text { Serum creatinine } \\
(\mathrm{mg} / \mathrm{L})\end{array}$ & $1.27 \pm 0.38$ & $1.5 \pm 0.75$ & SC \\
$\begin{array}{l}\text { Gender (Female/ } \\
\text { Male })\end{array}$ & $138 / 135$ & $35 / 15$ & CLR \\
$\begin{array}{l}\text { MTX dosage } \\
\left(\mathrm{g} / \mathrm{m}^{2}\right)\end{array}$ & 5 & 5 & GEN \\
\hline \hline
\end{tabular}

Table 2. Expected pharmacokinetic parameter estimates

\begin{tabular}{|c|c|c|c|c|c|}
\hline Parameter & Estimates & $\begin{array}{l}\text { Inter-individual } \\
\text { variability } \\
(\mathrm{CV} \%)\end{array}$ & $\begin{array}{l}\text { Inter-occasion } \\
\text { variability } \\
(\mathrm{CV} \%)\end{array}$ & $\begin{array}{l}\text { Relative } \\
\text { standard } \\
\text { error } \\
\text { (RSE \%) }\end{array}$ & $\begin{array}{l}\text { Confidence } \\
\text { intervals } \\
(95 \%)\end{array}$ \\
\hline CL (1/h) & 8.72 & 44 & 13.8 & 13 & [0.14-0.24] \\
\hline V1 (1) & 17.49 & 95 & - & 3.7 & {$[0.85-0.98]$} \\
\hline V2 (1) & 6.048 & 56 & - & 7.5 & {$[0.27-0.36]$} \\
\hline V3 (1) & 0.015 & 53 & - & 7.19 & {$[0.23-0.31]$} \\
\hline $\begin{array}{l}\text { Residual } \\
\text { variability }\end{array}$ & $\sigma$ & 75 & - & - & - \\
\hline
\end{tabular}


demonstrate that retained covariates contributed to an improvement of the fit of the population pharmacokinetic model, each covariate was deleted sequentially from the proposed final model (backward elimination) in order to confirm statistical significance $\left(\chi^{2}\right.$ test). If the objective function did not vary significantly, the relationship between the covariate and pharmacokinetic parameter was ignored. (Table 3) summarizes the data of the covariates.

Inter-occasion variability was investigated on CL, V1, and V2 and was only significant on CL.

Plots of model-population predicted versus population observed concentrations obtained from the final model based on individual and population parameter estimates are shown in Figs. 1a and 1b. Various statistical tests were carried out with a no significant outcome difference when the regression line of individual predicted concentrations versus observed concentration (slope $=0.63$, $\mathrm{SE}=0.03$; intercept $=1.56$ ) was compared to the reference line slope $=1$ and intercept $=0$ ); and the frequency of the distribution histogram of the normalized residuals was as expected (normal with zero mean and unitary variance). The vast majority of the weighted residuals laid within two units of perfect agreement and was symmetrically distributed around the zero ordinate (Fig. 1b).

Table 3. Forward selection model building summary

\begin{tabular}{lllll}
\hline \hline No. & Model* & OBJ & LLD** & $\begin{array}{l}\text { Signific- } \\
\text { ance*** }\end{array}$ \\
\hline 1 & Base Model & -919 & - & No \\
2 & 1+Age $\sim$ Cl & -919 & 0 & No \\
3 & 2+WT $\sim$ Cl & -1041 & 122 & Yes**** \\
4 & 3+GEN $\sim \mathrm{Cl}$ & -742 & -177 & No \\
5 & 4+CLR Cl & -1079 & 160 & Yes**** \\
6 & $5+\mathrm{Age} \sim \mathrm{V} 1$ & -956 & 37 & Yes**** \\
7 & 6+WT $\sim$ V1 & -919 & 0 & No \\
8 & 7+GEN $\sim \mathrm{V} 1$ & -919 & 0 & No \\
9 & $8+\mathrm{CLR} \sim \mathrm{V} 1$ & -919 & 0 & No \\
10 & $9+\mathrm{Age} \sim \mathrm{Q} 2$ & -736.34 & -183 & No \\
11 & $10+\mathrm{WT} \sim \mathrm{Q} 2$ & -897.22 & -22 & No \\
12 & $11+\mathrm{GEN} \sim \mathrm{Q} 2$ & -919 & 0 & No \\
13 & $12+\mathrm{Age} \sim \mathrm{Q} 3$ & -919 & 0 & No \\
14 & $13+\mathrm{WT} \sim \mathrm{Q} 3$ & -926 & 7 & Yes**** \\
15 & $14+\mathrm{GEN} \sim \mathrm{Q} 3$ & -919 & 0 & No \\
\hline
\end{tabular}

*=Model indicates sequential steps taken to achieve a full model. ' $\sim$ ' symbolizes 'as a linear function of'. Intercept terms are implicit; $* *=$ LLD is the change in objective function value obtained for the modelling step; $* * *=$ Significance is at the $\mathrm{P}<0.05$ level $(\mathrm{LLD}>3.84) ; * * * *=$ Covariate dropped in final model as the $95 \%$; confidence interval enclosed zero.
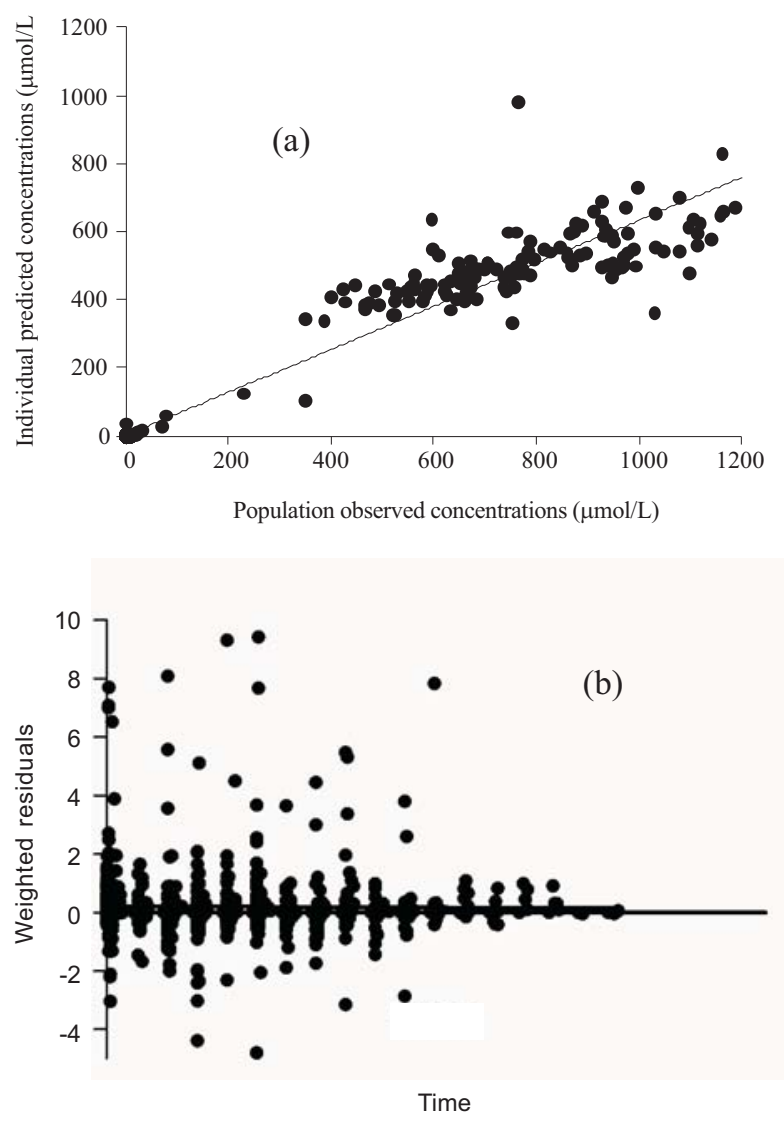

Fig. 1a-b. Model performance and diagnostic plots $(\mathrm{n}=273$ patients; 2582 MTX concentrations) a- Model populationindividual predicted versus populationobserved concentrations obtained with the final model based on population parameter estimates. b- Weighted residuals versus time.

Development of the full model. The final regression model was determined as follows;

Clearance:

$\left.\mathrm{CL}\left(1 \mathrm{~h}^{-1}\right)=(\theta 1+\mathrm{WT} * \theta 7)+(\theta 9 * \mathrm{CLR})\right) * \operatorname{EXP}(\operatorname{ETA}(1))$

Volume of distribution:

$\mathrm{V}_{\mathrm{C}}=\theta 2+\left(\mathrm{AGE}^{*} \theta 8\right) * \operatorname{EXP}(\mathrm{ETA}(2))$

Intercompartmental clearance:

$\mathrm{Q}\left(1 \mathrm{~h}^{-1}\right)=\theta 6^{*} \mathrm{WT}$

Table 4 displays the expected pharmacokinetic parameters estimates, additionally as estimated by NONMEM, intersubject coefficient of variation (CV) for the final model is presented.

To assess its predictive performances, the final model was used to predict concentrations in a new group of 
Table 4. Predictive performance of final model established in the validation group

\begin{tabular}{ll}
\hline \hline Final model & Error \\
\hline $4.88(2.73-7.02)$ & Mean prediction error (MPE) \\
$2.21(2.17-2.26)$ & Mean squared prediction error (MSPE) \\
$1.48(0.39-1.53)$ & Root mean squared prediction error (RMSE) \\
\hline \hline
\end{tabular}

patients. Table 4 summarizes the prediction error in the qualification group. The results show no bias, a good precision and indicate acceptable predictive performance.

Previous reports in the literature suggest that the disposition of methotrexate could be characterized by a two or three compartment model with first order elimination (Zhang et al., 2010; Colom et al., 2009; Aumente et al. 2006; Faltaos et al., 2006). In our population model was fitted to the present data. Model diagnostics in the form of residual plots and the log likelihood difference suggested that the two compartment model offered no improvement in the fit to the data while the three compartment model best fitted ours data. Therefore, subsequent modeling was implemented with a three compartment model.

In this analysis, mean values for clearance, and central volume of distribution, values CL, $8.72 \mathrm{lh}^{-1}$; V1, 17, 49 1 estimated by NONMEM were close to values reported in other population pharmacokinetic study as values reported by Plard et al. (2007) CL, $8.8 \mathrm{lh}^{-1} ; \mathrm{V} 1,17,3$ and by Rousseau et al. (2002) CL, $7.4 \mathrm{lh}^{-1}$; V1, 18,2 1.

Clearance increased with increasing renal filtration as assessed by creatinine clearance and increased with increasing body weight, presumably representing nonfiltration elimination. Statistically significant correlation was reported between creatinine clearance and total body clearance of methotrexate (Zhang et al., 2010; Oudoul et al., 1999; Monjanel et al., 1979). However, these relations were for one variable only and of poor predictive value. Combe et al. (1995) and Lafforgue et al. (1995) also reported a statistically significant correlation between methotrexate clearance and creatinine clearance.

More recently, Faltaos et al. (2006) in a population pharmacokinetic analysis reported a statistically significant positive correlation between creatinine clearance and methotrexate clearance. It was also observed that methotrexate clearance was inversely correlated to patient age, although the relationship was not as strong as with creatinine clearance (Colom et al., 2009; Aumente et al., 2006; Faltaos et al., 2006).,
Lafforgue et al. (1995) reported similar findings on the relationship between age, creatinine clearance and methotrexate clearance. However, in the full model, the parameter relating methotrexate clearance to age was not precisely estimated as evidenced by a $95 \%$ confidence interval enclosing zero. The clinical interpretation of such results might be that age related decreases in methotrexate clearance are adequately accounted for by incorporation of creatinine clearance assessments. Therefore, renal function monitoring is necessary in patients receiving methotrexate and adjusting appropriate dosage.

Volume of the central compartment was approximately 17.491 in this study population. This value is somewhat lower than that reported by Bressolle et al. 1996 (34.8 1) but similar (22.21) to that published by Zhang et al. (2010); Plard et al. (2007); Oudoul et al. (1999) and Sabot et al. 1995). Volume of the peripheral compartment increased with increasing body weight.

The population pharmacokinetics of methotrexate in children with ALL was determined using an extended least squares approach to nonlinear mixed effects modelling. Estimates of mean pharmacokinetic parameters correspond well with values previously reported in the literature. However, the population approach has provided estimates of intersubject variability and a method by which to explain some of this variability in terms of subject specific covariates. This can be seen in Table 4 . The population pharmacokinetics of methotrexate in children with ALL was well described by this investigation. Substantial interpatient variability was explained by incorporating patient specific data into regression equations predicting pharmacokinetic parameters and the clinical relevance and implications of the relationships have been presented.

\section{Conclusion}

The pharmacokinetic parameters of high dose methotrexate were accurately estimated. Creatinine clearance and weight influenced the high methotrexate clearance and age influenced volume distribution. Care should be taken in the choice of the dose regimen.

\section{References}

Aumente, D., Buelga, D.S., Lukas, J.C., Gomez, P., Torres, A., Garcia, M.J. 2006. Population pharmacokinetics of high-dose methotrexate in children with acute lymphoblastic leukemia. Clinical 
Pharmacokinetics, 45: 1227-1238.

Balloy, T., Desroches, M.C., Moussay, C., Merkadal, L., Fernandez, C. 2007. Modalités de prise en charge des intoxications aiguës par le méthotrexate haute dose. Journal de Pharmacie Clinique, 26: 253260.

Bresolle, F., Bologna, C., Edno, L., Bernard, J., Gomeni, R., Sany, J.C. Combe, B. 1996. A limited sampling method to estimate methotrexate pharmaco-kinetics in patients with rheumatoid arthritis using a Bayesian approach and the population data modelling program P-Pharm. European Journal of Clinical Pharmacology, 49: 285-292.

Colom, H., Farre, R., Soy, D., Peraire, C., Cendros, J.M., Pardo, N., Torrent, M., Domenech, J., Mangues, M.A. 2009. Population pharmacokinetics of high-dose methotrexate after intravenous administration in pediatric patients with osteosarcoma. Therapeutic Drug Monitoring, 31:76-85.

Combe, B., Edno, L., Lafforgue, P. 1995. Total and free methotrexate pharmacokinetics, with and without piroxicam, in rheumatoid arthritis. British Journal of Rheumatology, 34:421-428.

Dupuis, C., Mercier, C., Salas, S., Yang, C., Deville, J., Balti, M., Ciccolini, J., Iliadis, A., Baciuchka, M., Duffaud, F., Favre, R. 2006. Implication of clinical and paraclinical covariates in the pharmacokinetics of high dose MTX (HD-MTX) in patients with osteosarcoma. Journal of Clinical Oncology, 24: 120-122.

Falkenrodt, A., Manel, A.M., Suciu, S., Solbu, G., Tokaji, E., Otten, J., Philippe, N., Magaud, J.P. 1994. Immunological results of protocol 58881 from EORTC Children Leukemia Cooperative Group. Leukemia and Lymphoma, 13: 99-100.

Faltaos, D.W., Hulot, J.S., Urien, S., More, V., Kaloshi, G., Fermandez, C., Xuan, K.H., Leblond, V., Lechat, P. 2006. Population pharmacokinetic study of methotrexate in patients with lymphoid malignancy. Cancer Chemotherapy and Pharmacology, 58: 626633.

Lafforgue, P., Monjanel-Mouterde, S., Durand, A.,
Catalin, J., Acquaviva, P. C. 1995. Lack of correlation between pharmacokinetics and efficacy of low dose methotrexate in patients with reumathoid arthritis. The Journal of Rheumatology, 22: 844849.

Monjanel, S., Rigault, J.P., Cano, J.P., Carcassonne, Y., Favre, R. 1979. High-dose methotrexate: preliminary evaluation of a pharmacokinetic approach. Cancer Chemotherapy and Pharmacology, 3: 189-196.

Oudoul, F., Le guellec, C., Lamagnere, J.P., Breich, D., Saux, M.C., Autret-Leca, E. 1999. Prediction of methotrexate elimination after high dose infusion in children with acute lymphoblastic leukaemia using a population pharmacokinetic approach. Fundamental \& Clinical Pharmacology, 13: 595604.

Plard, C., Bressolle, F., Fakhoury, M., Zhang, D., Yacouben, K., Jacqz-Aigrain, E . 2007. A limited sampling strategy to estimate individual pharmacokinetic parameters of methotrexate in children with acute lymphoblastic leukaemia. Cancer Chemotherapy and Pharmacology, 60: 609-620.

Rousseau, A., Sabot, C., Delepine, N., Delepine, G., Debord, J., Lachatre, G. Marquet, P. 2002. Bayesian estimation of methotrexate pharmacokinetic parameters and area under the curve in children and young adults with localised osteosarcoma. Clinical Pharma-cokinetics, 41 : 1095-1104.

Sabot, C., Debord, J., Roullet, B., Marquet, P., Merle, L., Lacharte, G. 1995. Comparison of 2 - and 3-compartment models for the bayesian estimation of methotrexate pharmacokinetics. International Journal of Clinical Pharmacology and Therapeutics, 32: 164-169.

Sheiner, L.B., Ludden, T.M. 1992. Population pharmacokinetics/dynamics. Annual Review of Pharmacology and Toxicology, 32: 185-209.

Zhang, C., Zhai, S., Yang, L., Wu, H., Zhang, J., Ke, X. 2010. Population pharmacokinetic study of methotrexate in children with acute lymphoblastic leukemia. International Journal of Clinical Pharmacology and Therapeutics, 48: 11-21. 